\title{
Empowering Teachers for Innovations: The Case of Online Teacher Learning Communities
}

\author{
Onno De Jong \\ Karlstad University, Karlstad, Sweden; Utrecht University, Utrecht, The Netherlands \\ Email: o.dejong@uu.nl
}

Received 2012

\begin{abstract}
Implementing innovations in classrooms often evokes a variety of recurrent difficulties, especially feelings of resistance among experienced teachers. Modern teacher education aims at reducing their opposition by empowering these teachers for developing new knowledge, beliefs, and skills. A growing number of these teacher courses is designed as teacher learning communities (TLC-s). A specific category of them, online networks, is the scope of the present paper. Main values and attributes of these communities are addressed. This is followed by presenting some leading principles for designing TLC-s. Important principles are: (i) creating subcommunities within large-scale online networks, (ii) combining online activities with face-to-face meetings, and, (iii) facilitating more equality in online group participation. These principles are illustrated by examples of real practices. Finally, main conditions for successful new online TLC-s are presented. Prospects for advanced studies of practices of these communities are also given.
\end{abstract}

Keywords: Teacher Learning; Online Communities; Network Design Principles; Network Practices

\section{Introduction}

Education in many countries is strongly influenced by the growing demand from society to prepare students for a changing world in which they are able to actively participate in complex societal discussions and difficult societal decisions. Students also need to develop interpersonal skills and professional attitudes, such as being able to work in teams, to communicate effectively, to criticize constructively and to uphold ethical behavior. In responding to the many challenges of the changing world, education is developing a range of new ideas and practices for teaching and learning (De Jong, 2007). Although these responses vary from country to country, some are shared because of a common interest. Main innovations in current education are the implementation of inquiry-based and problem-based approaches that provide students opportunities for active learning, collaborative as well as self-directed, and for acquiring valuable knowledge and life skills required in their future careers and for further education. Another main innovation is the implementation of context-based approaches to bring new issues from society and technology into the classroom for developing critical thinking and enhancing the relationship between the world inside and outside school. Finally, a range of new technologies is introduced for supporting or transforming current educational practices, for instance, interactive software for students, interactive whiteboards for classroom discussions, computer-assisted instruction (CAI), and Web-based interactive learning environments.

Teachers are the most important factor for effectively and successfully implementing innovations in education. However, these implementations often evokes a variety of recurrent difficulties, especially feelings of resistance among experienced teachers. Modern teacher education aims at reducing this opposition by empowering these teachers for developing new knowledge, beliefs, and skills (Stolk, De Jong, Bulte \& Pilot,
2011). A growing number of these teacher courses is designed as networks. A specific category of them, online networks, is the scope of the present paper.

The structure of the paper is as follows. The next section deals with important factors that contribute to difficulties in implementing innovations in classrooms. Thereafter, main values and attributes of online teacher learning communities are addressed. This is followed by presenting some important leading principles for designing these networks. Finally, conditions for establishing new online networks are presented and prospects for advanced studies of online network practices are given. Note: the term 'teachers' in this paper does not refer to student teachers or beginning teachers but to experienced teachers.

\section{Difficulties in Implementing Innovations in Classrooms}

Difficulties in implementing innovations in classrooms can be caused by a complex of related factors. Some of the most important factors are given below.

Firstly, the hindering factor of the local school context of school, subject department, and available time, money and other resources. Success in implementing changes in teaching depends strongly on the culture of the school, especially responses of school management and attitudes of teacher peers (Jones, 1997). School management and teachers have to discuss and develop and discuss a joint vision on an innovative approach. However, the existing culture and discourse communities in many schools do not support a critical examination of teaching practices. Subject department meetings often provide few opportunities for meaningful reflection and professional growth. The availability of sufficient time is also important. It is well-known that many teachers express concerns about the amount of time it takes to incorporate innovations in their les- 
sons. Moreover, they often complain about a lack of money for appropriate technological equipment and facilities issues.

Secondly, the hindering factor of the experienced teachers themselves. Many of them show resistance towards implemented innovations and feel that they do not have enough expertise, for instance, insufficient knowledge and skills regarding appropriate new guidance for their students (Bliss, Askew, \& Macrae, 1996). Another example is regarding the use of new technologies. Many teachers, at least in the rich countries, possess personal computers, they connect them to internet at home, and have a positive attitude towards Internet use (Tekerek and Ercan (2012). However, it is well-known that many of them, especially older teachers, are not very familiar with the use of student computer software and similar ICT tools for education because they are not grown up with them. Dori and Barnea (1997) even found that teachers are not willing to introduce computer technologies in their lessons because of their fear off changes.

Thirdly, the hindering factor of the usual in-service courses. Smith and O'Day (1991) indicated that many of these courses often do not provide sufficient depth and scope to give teachers the experience to make major changes in their teaching approach. They do not focus very well on the (practical) needs of teachers. The usual courses do often not create many opportunities for meaningful interactions with peers or the teacher educator. For instance, Galanouli and Murphy (2004) investigated an ICT-competence teacher course and found that the course did improve teachers' confidence in using ICT, but that many teachers were not satisfied with the communication element, especially the use of discussion forums to deliver online training. Many teacher courses create largely short-lived changes in teachers' classroom strategies. In other words, the impact of these courses is often washed out quite soon through the lack of ongoing support.

In conclusion, simply dumping innovations in the school is, in itself, unlikely to transform teaching practices. Teachers need guidance and empowerment for engaging in productive discussions and innovative teaching. Their professional development should be embedded in teacher courses that are based on modern views on teacher learning.

\section{Teacher Learning Communities}

\section{Networks of Teachers}

Many modern courses for teacher professional development are influenced by socio-constructivist views on learning. They conceive teachers as active and reflective practitioners and create conditions for collaborative and self-directed learning. Several studies of effective attributes of these courses suggest that interaction among teachers is primary in facilitating teacher change processes (Connelly \& Clandinin, 1988; Day, 1987; Heller, Daehler, Wong, Shinohara, \& Miratrix, 2012). Discussing and sharing experiences with colleagues similarly engaged are very fruitful learning activities. Collegial talk possesses a strong motivational character. Structuring communication between teachers can contribute to help teachers interpret curriculum innovations and to provide a context that builds professional learning. This has stimulated the interest in establishing teacher courses that are designed as interactive networks: teacher learning communities (TLC-s).

Teacher learning communities are networks to which par- ticipants feel they belong and in which they feel accepted. The core group of a TLC consists of teachers but other groups can also be involved, for example, designers of networks and cur ricula, teacher educators, and, in case of use of modern technologies, ICT specialists. In the past decade, the number of TLC-s that use Internet for effective communication and collaboration has increased. These online communities provide attractive opportunities for teachers, for instance, they can decide from where and at what moment they communicate with members of the network. The communication can be synchronous, for instance the use of audio-chat and videoconferencing, or asynchronous, for instance the use of e-mails, discussion forums, and video sharing.

\section{Values of Online TLC-s}

The value of non-online TLC-s for teacher professional development has been specified by several scholars (Adams, 2000; Putman \& Borko, 2000; Wenger, 1998). In this paper, the most important of these values are adapted for online TLC-s. They are summarized in Figure 1.

Another important value of online TLC-s concerns the extent of interactions between participants of non-online in-service courses after the courses are finished. This value is illustrated by the following example. Fine (1993) reported about an online project, called Computer-assisted Writing Project (CAWP On-Line). This project functions as a follow-up of a course for improving the teaching of writing through computer-assisted instruction. The CAWP On-Line project aims at empowering teachers who had finished the course to become expert teachers who are able to encourage non-participating peers to try innovative teaching methods. For that purpose, a computer-based conference and related services are established that includes e-mail facilities, online discussion centers, and access to relevant online data bases and other resources. The study indicates that approximately half of the potential users did in fact become high users of this follow-up project. In conclusion, the given example suggests that a follow-up of in-service courses by online TLC-s can provide important and fruitful ongoing empowerment for teachers.

\section{Attributes of Online TLC-s}

Main attributes of non-online TLC-s have been addressed by Cochran-Smith and Lytle (1999) and McLaughlin and Talbert (2006). In this paper, the most important of these attributes are adapted for online TLC-s. They are summarized in Figure 2.

* Providing flexibility of time and location

* Reducing teachers' resistance to innovations

* Enhancing teachers' self-efficacy in new teaching

* Developing new expertise with peers

* Facilitating teachers' co-ownership of innovations

Figure 1.

Main values of online teacher learning communities.

* Collaboration in a supporting network

* Common interest in practice problems

* Commitment to network goals and efforts

* Try-out of new teaching strategies and materials

* Discussions and reflections on experiences

* Sharing of new expertise and best practices

Figure 2.

Main attributes of online teacher learning communities. 


\section{Online Communities: Design Principles and Practices}

Designs of online teacher learning communities are based on one or more principles. For instance, the principle of duration has an influence on the decision to design a short-termed or long-termed TLC, the principle of span functions as a base for deciding whether a TLC involves teachers who come from the same school or from different schools, and the principle of setting influences the decision to design a TLC that includes very loose arrangements or more formalized arrangements. In this section, some important leading design principles are more elaborated.

\section{Creating Subcommunities within Large-scale Online Communities}

Large-scale online TLC-s have the profit of providing a broad range of electronic resources and tools for developing and sharing expertise and experiences for (potential) use in own practice. However, they have the risk of evoking community-wide discussions that do not fulfill the specific needs of each teacher. This is illustrated by the following example.

Baek and Barab (2005) reported on a large online project, called the Inquiry Learning Forum (ILF) that had about 10,000 postings to ILF forums in the mid-2000s. This project provides science and math teachers a variety of tools for developing, sharing, and improving inquiry-based teaching practices. Teachers can virtually visit each others' classrooms through the viewing of online video vignettes. In these visits, teachers can get an overview of the lesson context, the lesson plans, and examples of student work. They can also hear or read the presenting teachers' reflections on the lesson. Teachers can be involved in online discussions with each other regarding the observed lesson and can pose questions to the presenting teacher. ILF has portal areas for obtaining help and feedback from teacher educators and technology experts, and there is a resource library for software, classroom tools, and relevant documents. One of the most interesting findings of their study shows that teachers who were very motivated regarding their professional development and acknowledged the importance of reflection lost a lot of interest when discussion sites and site contents were not directly related to their daily teaching. Because of this project outcome the design of ILF was partly restructured towards smaller networks within the larger project space. Each of these networks had the opportunity to work together on a particular topic of common interest. This change improved the involvement of teachers and stimulated their mutual discussions.

In conclusion, the example given suggests that the implementation of subcommunities within large-scale projects can be to be an effective way for fostering meaningful professional discussions and development.

\section{Combine Online Activities with Face-to-Face Meetings}

Teachers who communicate with other participants of a TLC by only using online facilities are often not very satisfied about this situation because they experience relative isolation despite the opportunities of online chats. As a consequence, it can reduce teacher involvement and can function as a hindrance to develop a coherent community with commitment to goals and efforts. However, combining online discourses with face-to- face meetings may contribute to foster productive TLC-s. Based on this idea, several online TLC-s were designed that intersperse online activities with face-to-face contacts between the participants. The following examples are an illustration.

Some time after the initial rollout of the ILF project (see preceding subsection), the decision of the management was to try to improve the quality of the project by implementing faceto-face workshops and other meetings in the project (Barab, MaKinster \& Scheckler, 2004). This brought together teachers who had previously interacted only online. This effort contributed to generating more meaningful interactions and reflections. The face-to-face components of the project were crucial to its success, but the online activities were also crucial, which led to a strong cooperation among participants .

Ruopp, Gal, Drayton and Pfister (1993) investigated a project, called LabNet, that focused on implementing an innovative (project-based) science teaching approach. The teachers interacted with each other from time to time in face-to-face small groups in their own regions but more often through computer conferencing. They also used conferencing for receiving empowerment from their teacher educators and scientists. The results show that the teachers' willingness to try out innovative teaching was increased. They even encouraged non-participating colleagues to explore the innovative teaching approach.

In conclusion, the examples given support the idea that the combination of online activities and face-to-face meetings can provide synergy for producing fruitful teacher professional development.

\section{Facilitating More Equality in Online Group Participation}

In many online TLC-s, the setting of goals, topics, and/or methods of communication is often strongly influenced by a specific group of participants: the experts. Their expertise regards areas as teacher education, network and curriculum unit design, and ICT systems. Many participating teachers have ambivalent feelings towards this situation. On the one hand, they feel that it is easy to follow the experts, on the other hand, they are adult learners and, for that reason, they like more self-directed learning. (Candy, 1991). To solve this 'agenda setting dilemma' (Richardson, 1992) it is important that teachers and experts of an online TLC share influence and contribute to network activities in an equal way. The implementation of this idea is illustrated by the following examples.

An important approach for creating more equality consists of neutralizing the dominant influence of experts by giving teachers the opportunity to propose their own topics as common themes of interest. In the context of the ILF project, Barab, MaKinster and Scheckler (2004) reported on teachers who shared a common interest, form a subcommunity, and brought in their own topics for discussion and further collaboration. It appeared that topics proposed by the teachers themselves were more popular than those introduced by system designers.

Another approach for facilitating more equality consists of developing equal participatory design activities. Rodrigues (2006) presented a study of an online community in which science teachers worked in teams with teacher educators and other experts to design resource materials involving various new technologies that are used by the teachers with their classes. The participants had face-to-face communication on a regular 
base and worked together online at other times through participants blogs and shared online spaces. As a result, teachers were effectively able to reform their practice in the intended way. The teachers appreciated the direct relevance of the tasks undertaken to their classroom teaching. This contributed to enhance their feeling of co-ownership of the innovative project and ownership of their personal professional development.

In conclusion, the examples given suggest that promoting more equality of teachers and experts can contribute to improved teachers' involvement in online TLC-s, and, for that reason, to a successful implementation of innovations.

\section{Towards Online Teacher Learning Communities for Tomorrow}

\section{Conditions for Successful New Online Communities}

From an analysis of the studies presented in the preceding section, it is possible to suggest main conditions for successful new online TLC-s. Some of these conditions are also valid for non-online TLC-s. For instance, the important conditions of setting clear community goals and desired outcomes, taking care that all participants hold or develop a similar perspective on teaching and learning, clearly evaluating the community processes and outcomes, and getting support from the local school context. Additional important conditions are more specific for online TLC-s. They are listed in Figure 3.

The first condition in the list regards the factor of available time. It takes considerable time for many teachers to become familiar with conducting discussions within an online environment. They need sufficient time to participate in community activities, to reflect on the own practice, and to see and understand how peers handle innovative teaching practices.

The second condition is regarding the factor of available resources. Teachers need to have access to computer-based resource materials and easily usable resource libraries of high quality that fit the goals of the community. Moreover, access to Web 2.0 networking tools can support e-portfolio communities and blogging can be used for promoting reflective discussions about fear and frustration in the process of change. It is clear that teachers need to have Internet-connected computers in their school or classroom. This is not a big problem in the rich industrialized countries. For example, in the USA, about $95 \%$ of the public schools were online at the end of the last century (US Department of Education, 2000). However, the availability of resources is a real big problem in many poor developing countries.

The third condition in the list regards the factor of trust and safety. Teachers who join an online TLC often express a fear of showing their thought and practices to unknown colleagues' examination in a semi-public space (Rodrigues, 2006). This can hinder trust needed for active participation. Establishing a faceto-face meeting at the beginning of an online project can contribute to foster feelings of psychological safety and acceptation.

\footnotetext{
* Sufficient time for online teacher participation

* Access to high-quality Internet-connected resources

* Culture of trust among the online participants

* Internal subcommunities in large-scale online projects

* Combination of online activities and face-to-face meetings

* Equality in online group participation
}

Figure 3.
Conditions for successful new online teacher learning communities.

The final three conditions regard factors that already have been addressed in the preceding section.

\section{Prospects for Advanced Studies of Online Community Practice}

The presence of online TLC-s is quite young in the field of in-service teacher education. As a consequence, not so many empirical-based studies have been undertaken to examine TLC-s practices. For developing more research-based online TLC-s it is important to set an 'Agenda of Research'. The following items can be the core of this agenda.

* Longitudinal studies that examine the impact of online TLC-s on teachers' knowledge, beliefs, and reflective activities;

* Long-term investigations of how online TLC-s empower teachers in developing new classroom practices;

* Research into factors that contribute to success or failure of online TLC-s;

* Investigations of the impact of online TLC-s on learning processes of participating experts.

Finally, a useful research strategy is provided by the designresearch approach (Cobb, Confrey, diSessa, Lehrer, \& Schauble, 2003). In the context of online TLC-s, this approach will focus on exploring the usefulness and consequences of course frameworks and related network programs. Both are optimized in several cycles of development and research, especially through a repeated process of implementation, testing, revision and retesting. In this context, educational researchers will also become participants of the networks under consideration. This will be another enhancement of online communities focusing on teacher professional development for tomorrow's education.

\section{REFERENCES}

Adams, J. E. (2000). Taking charge of curriculum: Teacher networks and curriculum implementation. New York: Teacher College Press.

Baek, R., \& Barab, S. A. (2005). A study of dynamic design dualities in a web-supported community of practice of teachers. Educational Technology \& Society, 8, 161-177.

Barab, S. A., MaKinster, J. G., \& Scheckler, R. (2004). Designing system dualities: Characterizing and online professional development community. In S. A. Barab, R. Kling, and J. H. Gray (Eds.), Designing for virtual communities in the service of learning (pp. 53-90). Cambridge, UK: Cambridge University Press.

Bliss, J., Askew, M., \& Macrae, S. (1996). Effective teaching and learning: scaffolding revisited. Oxford Review of Education, 22, 37-61.

Candy, P. C. (1991). Self-direction for lifelong learning. San Franscisco: Jossey Bass.

Cobb, P., Confrey, J., diSessa, A., Lehrer, R., \& Schauble, L. (2003). Design experiments in educational research. Educational Researcher, 32, 9-13.

Cochran-Smith, M., \& Lytle, S. (1999). Relationships of knowledge and practice: Teacher learning in communities. Review of Research in Education, 24, 249-305.

Connelly, F. M., \& Clandinin, D. J. (1988). Teachers as curriculum planners: Narratives of experience. New York: Carnegie Cooperation.

Day, C. (1987). Professional learning through collaborative inservice activity. In J. Smyth (Ed.), Educating teachers: Changing the nature of pedagogical knowledge (pp. 207-222). New York: Falmer Press.

De Jong, O. (2007). Trends in Western science curricula and science education research: a bird's eye view. Journal of Baltic Science Education, 6, 15-22.

Dori, Y. J., \& Barnea, N. (1997). In-service vhemistry teachers’ train- 
ing: The impact of introducting computer technology on teachers' attitudes and classroom implementation. International Journal of Science Education, 19, 577-592.

Fine, C. S. (1993). CAWP On-Line: Enhancing collacoration through technology. In G. Davies and B. Samways (Eds.), Teleteaching (pp. 239-248). Amsterdam: North Holland Publishers.

Galanouli, D. C., \& Murphy, A. (2004). Teachers' perceptions of the effectiveness of ICT-competence training. Computers and Education, 43, 63-79.

Heller, J., Daehler, K., Wong, N., Shinohara, M., \& Miratrix, L. (2012). Differential effects of three professional developments models on teacher knowledge and student achievement in elementary science. Journal of Research in Science Teaching, 49, 333-362.

Jones, D. (1997). A conceptual framework for studying the relevance of context to mathematics teachers' change. In E. Fennema and B. Nelson (Eds.), Mathematics Teachers in Transition (pp. 131-154). Mahwah, NJ: Erlbaum.

McLaughlin, M. W., \& Talbert, J. E. (2006). Building school-based teacher learning communities: Professional strategies to improve student achievement. New York: Teacher College Press.

Putman, R. T., \& Borko, H. (2000). What do new views of knowledge and thinking have to say about research on teacher learning? Educational Researcher, 29, 4-15.

Richardson, V. (1992). The agenda-setting dilemma in a constructivist staff development process. Teaching and Teacher Education, 8, 287-300.

Rodrigues, S. (2006). Pedagogic practice integrating primary science and elearning: The need for relevance, recognition, resource, reflection, readiness and risk. Technology, Pedagogy and Education, 15, 175-189.

Ruopp, R., Gal, S., Drayton, B., \& Pfister, A. (1993). LabNet: Towards a community of practice. Hillsdale, NJ: Lawrence Erlbaum.

Smith, M., \& O’Day, J. (1991). Systematic school reform. In S. Fuhrman and B. Malen (Eds.), The Politics of Curriculum and Testing (pp. 233-268). Philadelphia: Falmer Press.

Stolk, M., De Jong, O., Bulte, A., \& Pilot, A. (2011). Exploring a framework for professional development in curriculum innovation: Empowering teachers for designing context-based chemistry education. Research in Science Education, 41, 369-388.

Tekerek, M., \& Ercan, O. (2012). Analysis of teachers' attitude towards Internet use: Example of chemistry teachers. Creative Education, 3, 296-303.

US Department of Education. National Center for Educational Statistics (2000). Internet access in US public schools and classrooms:1994-1999. Washington DC: US Government Printing Office.

Wenger, E. (1998). Communities of practice: Learning, meaning, and identity. Cambridge, UK: Cambridge University Press. 